\title{
Frameless
}

Volume 1 | Issue 1

Article 21

November 2019

\section{The Design of Interplay Between Virtual Reality and Augmented Reality: A Case Study and Post Mortem of VRsus guARdian}

Tanat Boozayaangool

Rochester Institute of Technology, txb9038@rit.edu

Follow this and additional works at: https://scholarworks.rit.edu/frameless

\section{Recommended Citation}

Boozayaangool, Tanat (2019) "The Design of Interplay Between Virtual Reality and Augmented Reality: A Case Study and Post Mortem of VRsus guARdian," Frameless: Vol. 1: Iss. 1, Article 21.

DOI: 10.14448/Frameless.01.008

Available at: https://scholarworks.rit.edu/frameless/vol1/iss1/21

This Article is brought to you for free and open access by RIT Scholar Works. It has been accepted for inclusion in Frameless by an authorized editor of RIT Scholar Works. For more information, please contact ritscholarworks@rit.edu. 


\title{
FRAMELESS
}

The Design of Interplay Between

Virtual Reality and Augmented Reality:

A Case Study and Post Mortem of VRsus guARdian

\author{
Tanat Boozayaangool \\ School of Interactive Games and Media \\ Rochester Institute of Technology
}

\begin{abstract}
VRsus guARdian is the result of an amalgamation of two different gameplay mediums that challenged the gap separating different mixed reality platforms, namely virtual reality (VR) and augmented reality (AR). The game utilized each medium's approach towards immersion as a design principle in building a natural, asymmetric play for both players. The game also constructed a compelling fantasy atop each medium's unique interactive capability to build a dynamic narrative. Lastly, the cross-platform nature of VRsus guARdian caused the game to be highly dependent upon Unity, a game engine with highly accessible, platformagnostic development capabilities.
\end{abstract}

Keywords: virtual reality, augmented reality, asymmetric play 


\section{INTRODUCTION}

VRsus guARdian is a cat-and-mouse chase that was built to explore the interplay between Virtual Reality (VR) and Augmented Reality (AR). One player utilizes an AR-enabled, mobile device and plays the role of the guardian, while the other equips a VR headset and plays the role of a tiny infiltrator. The AR device is used to scan the room and build out a virtual representation to bridge the experience between the VR and AR player. The AR player is then tasked with searching and eliminating the trespasser, while the VR player must strategically use their hookshot to maneuver through the environment and steal the world's relics.

\section{DESIGN APPROACH IN VRSUS guARdian}

The core principles driving VRsus guARdian's asymmetric gameplay was derived from the different ways in which VR and AR builds a sense of immersion for its participant. While VR experiences generally remove the participant from their current environment and immerse them with a unique reality, AR experiences tend to instead visually augment the participant's current surroundings.

With inspiration from games such as Sprint Vector and Windlands, an immersive, natural control scheme became the centerpiece of the VR player's design. As speed and momentum became a very important aspect of the experience, the hook shot was introduced to provide the VR player with vertical maneuverability within the foreign world.

The AR experience, on the other hand, was designed around uplifting a familiar play with an engaging narrative. By juxtaposing cartoon-inspired game models on top of a realistic, familiar environment, the theme of invasion surfaced itself as an assessible narrative for the AR experience. Furthermore, the social interactions throughout the experience and the novel usage of technology help enhance the gameplay of moving around the room and searching for a virtual invader.

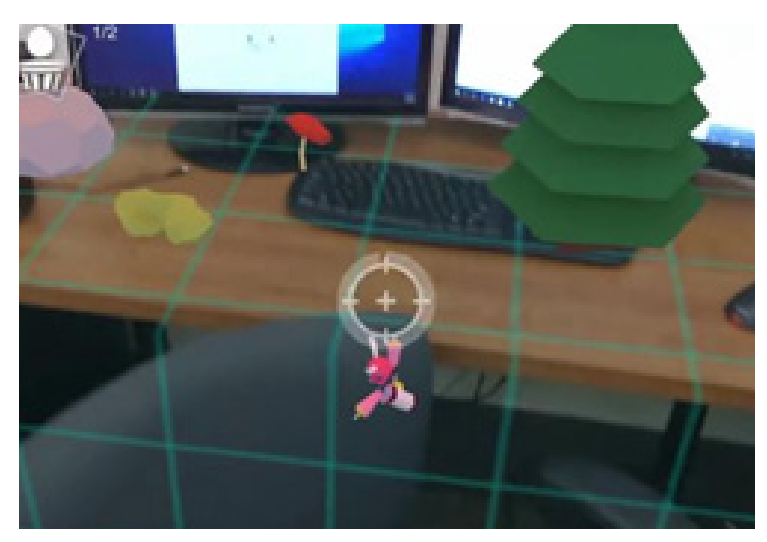

Fig. 1. VRsus guArdian screenshot taken from the AR player's perspective 
Vol. 1, No. 1 - 2019

Frameless

\section{FRAMING THE EXPERIENCE IN VRsus guARdian}

Another factor that influenced the overall experience of VRsus guARdian was the way in which the game was framed for each player. Unlike typical gaming experiences where motion is often simulated through a static monitor, VRsus guARdian fully takes advantage of the dynamicity in which the player's perspectives are framed.

In the VR experience, the player is often forced to look upwards to plan a route through the higher platforms, while evading the line of sight of the AR player. When the VR player eventually moves up onto one of the higher platforms, the shift in camera angle helps build a heroic narrative onto the VR player's journey throughout this environment. Meanwhile, the obstacles around the world often forces the AR player to assume different, intricate angles to accurately identify the location of the virtual intruder. This helps frames the narrative of the AR player as a powerful being limited by their lack of agility.

\section{GAME ENGINES AND THE DIRECTION OF VR/AR}

Lastly, the development of VRsus guARdian was greatly influenced by the design of Unity and the available tools in Unity development. As this game was cross-platform by its nature, Unity became the least costly choice in developing the game. However, features such as advanced pattern recognition and image processing became too costly as Unity and its AR capabilities were not natively designed to support such features. 